\title{
Ethnographie als Interaktionsprozess - Zur ethnographischen Untersuchung hybrider Lernorte
}

\author{
Karola Cafantaris
}

Eingegangen: 19. Dezember 2020 / Überarbeitet: 20. Januar 2021 / Angenommen: 10. Februar 2021 /

Online publiziert: 7. April 2021

(C) Der/die Autor(en) 2021

Zusammenfassung Der Beitrag thematisiert die ethnographische Erforschung hybrider Lernorte innerhalb der Erwachsenenbildung. Es werden forschungsbezogene Merkmale hybrider Lernsettings herausgestellt und die Herausforderungen und Stärken der ethnographischen Forschungsmethode für die Erschließung hybrider Lernsettings hervorgehoben. Dabei werden insbesondere die subjektiven Bezugnahmen der Forschenden auf das Untersuchungsfeld und die dabei entstehenden interaktionalen Prozesse fokussiert. Indem der ethnographische Forschungsprozess als Interaktion rekonstruiert wird, soll ein Beitrag zur Methodendiskussion innerhalb der Erwachsenenbildung geleistet und damit zugleich darauf gezielt werden, weitere Möglichkeiten der Erforschung hybrider Lernsettings auszuloten.

Schlüsselwörter Ethnographie · Qualitative Forschungsmethoden · Erforschung hybrider Lernorte $\cdot$ Erwachsenenbildung $\cdot$ Beobachtung $\cdot$ Informelles Lernen 


\title{
Ethnography as a process of interaction-ethnographic fieldwork in a hybrid place of learning
}

\begin{abstract}
The paper addresses the ethnographic research of hybrid learning settings within adult education. Research-related characteristics of hybrid learning settings are highlighted and the challenges and strengths of the ethnographic research method for opening up hybridised learning settings are emphasised. In particular, the subjective references of the researchers to the field of study and the interactional processes that emerge in the process are focused on. By reconstructing the ethnographic research process as an interaction, a contribution to the discussion of methods within adult education will be made and further possibilities for researching hybrid learning settings will be explored.
\end{abstract}

Keywords Ethnographical research · Qualitative research methods · Hybrid learning sites · Adult education · Informal learning

\section{Einleitung}

Die Entstehung neuer Lern- und Bildungsangebote stellt in der modernen Gesellschaft längst kein Novum mehr dar. Neben den traditionellen Bildungseinrichtungen, die selbst auch vermehrt Teil dieser Modernisierungs- und Diffusionsprozesse werden, bilden sich vielfältige Lern- und Bildungsorte heraus, die in einem traditionellen pädagogischen Vokabular, etwa mit den Begriffen „Erziehung“, „Bildung“ oder „Unterricht“, kaum mehr zu beschreiben sind. Aufgrund der Hybridisierung pädagogischer Institutionen, der Pädagogisierung verschiedener Lebensbereiche und der gleichzeitigen Vermehrung informeller pädagogischer Struktur- und Vermittlungsmuster beobachtet die Erziehungswissenschaft schon seit Längerem eine „Universalisierung des Pädagogischen“ (vgl. Lüders et al. 2004). Lüders, Kade und Hornstein (ebd.) sehen eine stetige Expansion pädagogischer Denk- und Handlungsstrukturen, die sich in nahezu allen Teilen der Gesellschaft zeigt und eine Allgegenwärtigkeit des Pädagogischen diagnostizieren lässt. Die Pädagogik sei noch nie ,so allgegenwärtig“ gewesen und werde heutzutage ,,als unversiegbare Quelle zur Lösung aller nur denkbaren Probleme herbeizitiert und gefordert" (ebd., S. 226). Der Strukturwandel der Bildungslandschaft und die Zunahme neuer Angebotstypen werden durch die sogenannte Entgrenzungsthese mit einem damit einhergehend modifizierten Blick der Wissenschaft untersucht, der ebenso eine daran angebundene Anpassung des Objektbereichs, auf den die Wissenschaft blicke, mit sich bringe (vgl. Seitter 2000, S. 81). Laut Dinkelaker (2018) würden Beschreibungen bedeutsam, ,die es erlauben, einen Überblick über die Pluralität von Bildungssituationen zu gewinnen“ (ebd., S. 16). Die Erforschung der Universalisierung des Pädagogischen erfordert demzufolge neben der theoretischen Auseinandersetzung mit dem Lernen im Erwachsenenalter (vgl. dazu Dinkelaker 2018) ebenfalls methodologische Neubestimmungen und Positionierungen innerhalb der erziehungswissenschaftlichen Forschung.

Die Ethnographie hat sich in den letzten Jahren als ,zentrale Strategie empirischer Forschung etabliert“ (Breidenstein 2017, S. 10). Vor allem innerhalb der qualitativen 
erziehungswissenschaftlichen Forschung gewinnt die Ethnographie zunehmend an Bedeutung (vgl. Heinzel et al. 2010; Cloos und Thole 2006). Dabei umschließen die Forschungsbereiche formalisierte Bildungssettings, wie die Schule (vgl. Wiesemann 2010; Heinzel 2010), sozialpädagogische Felder (vgl. Hünersdorf 2009), Bereiche der Kindheitsforschung (vgl. Bock 2010) und auch das Feld der Kindertageseinrichtungen (vgl. Bollig 2010). Auch die Erwachsenenbildung blickt auf einige ethnographisch verortete Arbeiten, die sich von formalisierten pädagogischen Feldern, wie etwa die der sozialpädagogischen Einrichtung (u. a. Hünersdorf 2009), über die Erforschung non-formaler Bildungsorte, wie des Vereinswesens (u.a. Seitter 1999) bis hin zu informellen Orten des Lernens von Erwachsenen (u. a. Wilde 2015), erstrecken. Thole et al. (2010) konstatieren, dass sich im „Kontrast zu anderen Forschungstraditionen [...] erst in letzter Zeit ein kontinuierlicher Diskussionszusammenhang zur methodischen Konsolidierung der Ethnographie herauszubilden scheint" (ebd., S. 12). Auf diese Forschungslücke Bezug nehmend, befasst sich der vorliegende Beitrag mit einer weiterführenden methodologischen Verankerung rund um die Frage der ethnographischen Erschließung eines hybriden Lernorts. ${ }^{1}$ Es wird zunächst die Bestimmbarkeit von hybriden Lehr-/Lernsettings untersucht (2), um daran anschließend die Besonderheiten einer ethnographischen Forschungsperspektive auf hybride Lernsettings herauszustellen (3). Daraufhin wird die Subjektivität im ethnographischen Forschungsprozess (4) als spezifische Haltung innerhalb der Forschung expliziert, um diese anhand eines empirischen Beispiels weiterführend auszuloten und sie einer abschließenden Diskussion (5) zu unterziehen.

\section{Zur Erforschung hybrider Lehr-/Lernsettings}

Empirische Untersuchungen in der Erziehungswissenschaft erkennen unter dem Gesichtspunkt einer Ausdifferenzierung von traditionell-institutionalisierten Lernorten neue Formen der Hervorbringung von Lernorten, die unter dem Stichwort des „Informellen" (vgl. Wischmann 2017) gefasst werden. Lernen und Bildung sind längst nicht mehr ausschließlich in den klassischen Bildungsinstitutionen verortet, sondern finden über die Lebens- und Altersspannen hinweg an unterschiedlichen Orten und unter unterschiedlich starker pädagogischer Leitung statt. Damit verbunden hat die Rede vom informellen Lernen und von informellen Bildungsorten Konjunktur erfahren und zu erziehungswissenschaftlichen wie auch bildungspolitischen Systematisierungen geführt (vgl. ebd.). Eine Allgemeingültigkeit des Informellen sei dabei, so Wischmann (ebd.), nicht feststellbar, so könne ,informelles Lernen immer nur in Beziehung zu formellem Lernen verstanden werden" und sei deshalb „kontext- und situationsgebunden“ (ebd., S. 60). Für die pädagogische Praxis hat dies zur Folge, dass es sogenannten hybriden Lernorten (Seitter 2011) zuweilen

\footnotetext{
${ }^{1}$ Die vorliegenden Überlegungen wurden in meiner Dissertation und dem daraus entstandenen Buch „Umgang mit Wissen in der Kulturarbeit für Erwachsene. Pädagogische Kommunikation in hybriden Settings der Erwachsenenbildung" (Cafantaris 2020) mit dem Schwerpunkt der Rekonstruktion von Kommunikationsformaten dargelegt und diskutiert. Als Vertiefung dessen dient dieser Artikel, der sich der in der Studie bereits angelegten und hier einer weiteren Reflexion unterzogenen ethnographischen Forschungshaltung widmet.
} 
an organisatorischen und didaktischen Grundlegungen und Strukturierungen fehlt, gleichzeitig jedoch Äquivalente für diese gefunden werden können. So stehen sich etwa in der informellen Erwachsenenbildung einerseits pädagogische Strukturierungsmomente und andererseits Freiwilligkeit und programmatische Offenheit gegenüber. Forschung zu Hybridität in der Erwachsenenbildung bewegt sich dementsprechend innerhalb der Spannung von informellen Zusammenkünften einerseits und formalisierter pädagogischer Formbildungen andererseits. Um diese pädagogischen Formbildungen $\mathrm{zu}$ untersuchen, treten dann vor allem empirische Forschungsdesigns in den Fokus, die der Frage nachgehen, wie organisatorische und interaktionale Bezugnahmen innerhalb des Untersuchungsfelds vollzogen werden und damit das Verhältnis von Diffusion und Stabilisierung austariert wird. Forschungen, die konkrete Interaktionen in den Blick nehmen, um der Frage nach hybriden pädagogischen Formbildungen nachzugehen, bleiben zuweilen jedoch eher die Ausnahme in der Erwachsenenbildungsforschung (vgl. Wischmann 2017). Folgt man Wischmann (ebd.), seien es ,weit weniger empirische Studien zum informellen Lernen Erwachsener“, als es „Expertisen nahelegen“ (ebd., S. 59). Außerdem „sei es auch weit unumstrittener, wie informelles Lernen definiert, differenziert und damit auch operationalisiert werden“ könne, als der ,politische[] Diskurs [es] suggeriert“ (ebd.). Gut belegt zu sein scheine ,lediglich, dass informelles Lernen (im Sinne nicht institutionell gerahmter oder curricular intendierter Lernprozesse) von großer Bedeutung ist, wie internationale Studien seit den 1970er-Jahren“(ebd.) zeigten. Dies berücksichtigend wird in den folgenden Abschnitten nachgezeichnet, wie sich hybride von formalisierten Lernsettings unterscheiden. Bedeutsam hierfür erscheinen vor allem die Auseinandersetzung mit der Frage nach den pädagogischen Formbildungen und deren forschungsbezogene Operationalisierung in Bezug auf die Untersuchung von hybriden Lernsettings (vgl. Dinkelaker 2008).

\subsection{Wie unterscheiden sich hybride von nicht hybriden Lernsettings?}

Gemäß der Systematisierung von Dinkelaker (2008) geht die Erziehungswissenschaft und Pädagogik von ,reinen Strukturen“ (ebd., S. 20) bezüglich der Ausgestaltung von Orten des Umgangs mit Wissen aus. So beschreibt er, dass ,solche Konzepte von getrennten Bereichen des Lernens und der Anwendung von Wissen lebensphasen- oder organisationsorientiert gedacht" würden (ebd.). Diese stark dichotome Trennung von formalen und nichtformalisierten Lern- und Bildungssettings würde jedoch zunehmend brüchig und es seien vermehrt ,Versuche zu beobachten, diese scharfe Trennung zu überwinden“ (ebd.). Dazu verhelfe in hohem Maße die Institutionalisierung des lebenslangen Lernens.

Mit dem Konzept des lebenslangen Lernens wird die Durchdringung von Lernen und Anwendung sogar zum Prinzip erhoben. Es setzt sich dezidiert von einer Vorstellung der Begrenzung von Lernzeiten und Lernorten ab (vgl. Fauré et al. 1973) (Dinkelaker 2008, S. 20).

Die in diesem Zitat beschriebene Wandlung von Begrenzung zu lose strukturierten pädagogischen Settings sowie der damit verbundene Umgang mit Wissen und Lernen zeigt sich bedeutsam im Begriff des informellen Lernens (vgl. dazu auch 
Wischmann 2017), der den Diskurs um hybride Lernorte entscheidend mitbestimmt (vgl. Dinkelaker 2008, 2018). Informelles Lernen bezeichnet demzufolge „Formen der Kombination von Lernen und Anwendung“, die als ,zentraler Bestandteil eines alle Lebensbereiche umfassenden lebenslangen Lernens" (Dinkelaker 2008, S. 20) beschrieben werden. Es handle sich um eine Form des ,individuellen Lernens“, die sich als ,erfahrungsbasierter und selbstgesteuerter Prozess“ (ebd.) versteht. Im Gegensatz zu formalisierten Lernsettings, wie sie etwa die Schule darstellt, vermischten sich in hybriden Lernorten ,unterschiedliche Formen des Umgangs mit Wissen bzw. Nicht-Wissen“ (ebd., S. 23). So würde in hybriden Settings ,,das Lernen und Nicht-Lernen der Beteiligten“ nicht ,aus dem sozialen Umgang mit Wissen ausgeblendet“, sondern es würde ,zum Gegenstand der Kommunikation“ gemacht (ebd.). Dabei würde das Bezugsproblem bearbeitet, ,dass die Interaktion einerseits ein fortbestehendes Wissen der Beteiligten“ voraussetze, andererseits jedoch „die Veränderung eben dieses Wissens zum Thema" (ebd.) habe.

In hybriden Settings werden Erwachsene zugleich als fortgesetzt kompetent und als lernend begriffen, als stabil und sich verändernd, als handlungsfähig und korrekturbedürftig. Obwohl es paradox ist, das Wissen einer Person zugleich als fest vorauszusetzen und als veränderlich zu behandeln, kann man kommunikative Prozesse beobachten, in denen dies dennoch geschieht (ebd.).

Die hier umrissenen Überlegungen zu hybriden Lernsettings machen deutlich, dass sich im Zuge der Diffundierung pädagogischer Lern- und Bildungsorte komplexe Verhältnisbestimmungen ergeben, deren Erforschung geeignete Methoden und theoretische Rückbindungen erfordert. Der Vorteil einer qualitativ orientierten Forschungsperspektive liegt in der Möglichkeit, durch spezifische Einblicke in pädagogische Felder die jeweiligen Eigenheiten und Bezugsprobleme zur Darstellung zu bringen. In der Rückbindung an den theoretischen Diskurs liegt die besondere Entfaltungskraft qualitativer Forschung. Wie im Folgenden dargestellt werden soll, erfordert die Untersuchung von hybriden Settings eine Perspektive, die die Unterscheidung von anwendungszentrierter und lernzentrierter Ausrichtung in den Blick zu nehmen vermag. So kann davon ausgegangen werden, dass die Erforschung hybrider Lernorte insbesondere die Erfahrungen der beteiligten Akteure eines Settings fokussiert (vgl. ebd.).

\subsection{Wie lässt sich die Erforschung hybrider Lernsettings charakterisieren?}

Davon ausgehend, dass sich in hybriden Lernsettings neben aneignungsbezogener Interaktion auch andere Interaktionsformen wie Geselligkeit, inhaltlicher Austausch oder Beratung vollziehen (vgl. Dinkelaker 2008), in denen ,,Verbindungen aus Sequenzen der Kommunikation von Lernen“ und des „Umgangs mit (Nicht-) Wissenszuschreibungen“ (ebd., S. 209) stattfinden, stellt sich erstens die Frage, unter welchen Bedingungen solche Lernsettings untersucht werden können, um möglichst das Changieren zwischen Formalisierung und Hybridisierung feststellen zu können. Die Unterscheidung von nichthybriden und hybriden Lernsettings spiegelt sich dahingehend auch in der Erforschung der jeweiligen Lernorte, da diese eine spezifische Offenheit gegenüber der eigenen Erfahrung innerhalb des Settings möglich machen. 
Stellt etwa die Erforschung des Schulunterrichts zumindest über das Vorhandensein von Lehr- und Stundenplänen eine in gewissem Maße erwartbare thematische Ausrichtung des Untersuchungsfeldes dar, wird dies in hybriden Lernsettings mit steigender Ungewissheit zunehmend offen und unsicher. In den Blick gerät damit vor allem die Verschränkung des sozialen Umgangs mit Lernen und der Anwendungs- und Aushandlungsformen von (Nicht-)Wissen (vgl. ebd., S. 20).

Die in hybriden Lernsettings institutionalisierten Muster des Umgangs mit (Nicht-)Wissen ermöglichen es, Lernen in Kontexten zum Thema zu machen, deren Funktion nicht auf den Umgang mit Lernen beschränkt ist. Deswegen kann die Untersuchung hybrider Lernsettings einen bedeutsamen Aspekt des informellen Lernens der empirischen Beobachtung zugänglich machen und damit zu einem differenzierteren Verständnis von Phänomenen der Entgrenzung des Pädagogischen beitragen (ebd.).

Laut Dinkelaker ist es für die Untersuchung hybrider Settings bedeutsam, Lernen als „sozial konstituiertes Phänomen“ (ebd.) zu verstehen. Dieser soziale Umgang mit Lernen zeichne sich ,gerade dadurch aus, dass in ihm auf das prinzipiell unbeobachtbare individuell-psychische Lernen immer nur verwiesen“ (ebd.) werden könne.

Der in diesem Beitrag verfolgte Ansatz sieht folglich ein interaktional hergestelltes Verständnis des Umgangs mit Wissen in hybriden Lernsettings vor. Dinkelaker folgend, wird Lernen dabei nicht als Versuch ,der absichtsvollen Einwirkung auf Lernen“ gefasst, sondern vielmehr, ,als Verfahren ihrer Repräsentation“ (ebd., S. 20).

\section{Die ethnographische Erforschung hybrider Lernorte}

Jede human- und sozialwissenschaftliche Forschung sei, so Rolf Lindner (1981) in seinem vielrezipierten Text Die Angst des Forschers vor dem Feld. Überlegungen zur teilnehmenden Beobachtung als Interaktionsproze $\beta$, eine spezifische Form sozialer Interaktion. Im Feld würde die Reziprozität zwischen Beobachtenden und Beobachtetem ,zum zentralen methodologischen Problem teilnehmender Beobachtung“ (ebd., S. 51). Und auch heutzutage wird das Verhältnis von Forschung und Feld methodologisch diskutiert. So ist die Frage nach wie vor aktuell, wie Forschende das von ihnen untersuchte Feld durch ihre Anwesenheit beeinflussen. BühlerNiederberger (2010) verweist deshalb darauf, ,die Frage nach der eigenen Identität nicht unabhängig von den Ergebnissen“ (ebd., S. 271) der Forschung zu stellen. Insgesamt sei es nicht nur eine Frage nach den Instrumenten, ,die damit in der Anfangs- und Feldphase zu stellen wäre, sondern auch eine Frage, die bei der abschließenden Reflexion der Ergebnisse dringlich wird, bei der es darum geht, die unterschiedlichen Perspektiven zu kontrastieren“ (ebd.). Das damit verbundene Bezugsproblem kann zunächst auf einer trivial anmutenden ersten Ebene wie folgt zusammengefasst werden: Forschende beeinflussen das von ihnen untersuchte Feld, genauso wie das Feld die Forschenden und damit auch die Ergebnisse der Forschung nicht unbeeinflusst lässt. Das methodologisch vorgegebene Fremd-Machen (vgl. Amann und Hirschauer 1997) der eigenen Kultur ist deshalb nur unter Vor- 
behalt einlösbar. Vor allem in länger angelegten Forschungsprozessen werden die Forschenden in ihrer Rolle zu Kooperationspartnerinnen, Verbündeten, aber auch Mitgliedern der Institution, in der sie forschen. Spätestens wenn es zu Ansprachen der Forschenden im Feld kommt, können diese nicht anders, als den interaktionalen Bezugnahmen nachzukommen und mit dem Feld zu interagieren. Die sich daran anschließende und längst nicht mehr trivial anmutende Frage lautet dann, wie das Feld und die Feldbeobachtung sich gegenseitig bedingen und eine gemeinsame Hervorbringungspraxis sozialer Wirklichkeit entsteht. Daran anknüpfend werden in den folgenden Ausführungen die Prinzipien der ethnographischen Forschungshaltung skizziert und auf das Verhältnis von Subjektivität und Forschungsprozess hin befragt. Es soll erläutert werden, wie sich die interaktionale Beziehung im ethnographischen Forschungsprozess niederschlägt, welche Bedeutung dem Eintritt ins Feld (vgl. Wolff 2000) ethnographisch zugeschrieben werden kann und wie sich diese Überlegungen in der konkreten Forschungspraxis (teilnehmende Beobachtung und Beobachtungsprotokoll) widerspiegeln.

\subsection{Zur ethnographischen Forschungshaltung}

Ihren Ursprung hat die ethnographische Forschungsmethode vornehmlich in der Erforschung sogenannter ,fremder“ Kulturen (vgl. Geertz 1987). Ihr geht es darum, durch das Eintauchen in einen kulturell fremden Kontext in Form einer teilnehmenden Beobachtung die Logik des untersuchten Feldes zu erkunden und zu erforschen. Die Methode verlangt von den Forschenden die körperliche Anwesenheit: An ihr manifestiert sich die Idee, in der stellvertretenden Aneignung des Gesehenen und des Erlebten etwas über die Erfahrungen, Gefühle und Handlungslogiken der Akteure eines Untersuchungsfeldes zu erfahren. Die stellvertretende Aneignung bezeichnet hierbei den Prozess des Erlebens der lokalen Situation, wie sie von der oder dem Forschenden im Untersuchungsfeld vorgefunden wird. Anders als bei der Analyse von Transkripten oder Videoaufzeichnungen wird bei der teilnehmenden Beobachtung die Anwesenheit in der Untersuchungssituation durch die oder den Forschenden zur methodologischen Prämisse erhoben, die den Analyseprozess entscheidend mitbestimmt. Ein anschauliches Beispiel für die Betonung der stellvertretenden Aneignung der lokalen Logiken des Feldes durch die Forschenden wird in Clifford Geertz' Klassiker Dichte Beschreibung. Beiträge zum Verstehen kultureller Systeme (1987) präsentiert: Dort beschreibt Geertz, wie er als Forschender in einem balinesischen Dorf eine ethnologische Untersuchung durchführen möchte, am Anfang seines Aufenthalts jedoch bei den Bewohnern des zu untersuchenden Dorfes zunächst keine Beachtung findet.

Wir kamen von Berufs wegen als Eindringlinge, und die Balinesen behandelten uns in einer Weise, die anscheinend ihren Gewohnheiten gegenüber Leuten entsprach, die nicht ihrer Lebenswelt angehörten und die sich doch aufdrängten, nämlich so, als ob wir nicht vorhanden wären. Für sie, und in einem gewissen Grade für uns selber, waren wir Unpersonen, Phantome, Unsichtbare (ebd., S. 202). 
Erst als der Forscher und seine Frau während des Beobachtens eines unerlaubten Hahnenkampfs mitsamt den lokalen Besuchern des Hahnenkampfes selbst in die Bredouille einer dem Hahnenkampf geltenden polizeilichen Razzia gerieten und daraufhin von einem Bewohner des Dorfes freundlich aufgenommen und gegenüber der Polizei verteidigt wurden, erhielt der Forscher durch das gemeinsame Erlebnis Zugang zur Dorfgemeinschaft.

„Dies war der Wendepunkt in unserer Beziehung zur Gemeinschaft und wir waren im wahrsten Sinne des Wortes, in'. Das gesamte Dorf tat sich uns auf, wahrscheinlich mehr und mit Sicherheit schneller, als das sonst der Fall gewesen wäre [...]“" (ebd., S. 207).

Ebenso wichtig an dem von Geertz beschriebenen Ereignis ist dessen Bedeutung für die darauffolgende Erforschung des balinesischen Dorfes und insbesondere des Hahnenkampfes als „,eep play“. So beschreibt er, durch diese Form des Eintritts in das Forschungsfeld einen wichtigen Untersuchungsgegenstand gefunden $\mathrm{zu}$ haben, der ihn einen Großteil seines Aufenthalts in dem Dorf beschäftigt hat (vgl. ebd., S. 202-260). So stellt der gelungene Eintritt ins Feld nicht nur den tatsächlichen Zugang zum Forschungsfeld dar, sondern verweist auch auf den ethnographischen Untersuchungsstil, der sich in der offenen Forschungshaltung des Forschenden zeigt. Es geht der Ethnographie darum, sich dem Untersuchungsfeld unvoreingenommen anzunähern und dieses ausgestattet mit einem befremdeten Blick zu erforschen (vgl. Amann und Hirschauer 1997). Das Programm der „Befremdung der eigenen Kultur“ (ebd.) sieht vor, vor allem bereits bekannten Orten mit einer künstlich eingenommenen Distanz zu begegnen. In der Untersuchung bekannter Untersuchungsfelder liegt der Reiz darin, die Alltäglichkeit des Bekannten methodisch kontrolliert wieder auf Distanz zu bringen, um sich dem Feld und den darin vorzufindenden kulturellen Logiken unvoreingenommen nähern zu können. Der subjektive Charakter der Erfahrungen und Wahrnehmungen der Forschenden wird in der Ethnographie im Unterschied zu technisierten Forschungsmethoden demzufolge gerade nicht eliminiert, sondern kann und soll produktiv fruchtbar gemacht werden. Dies macht einen Rückgriff ,,auf das umfassende Spektrum kognitiver Modi wie Zuhören, Fragen, Lesen, das aktive Intervenieren im Feld und die reflexive Introspektion“ (Thomas 2019, S. 3) notwendig. Die Forschenden etablieren sich damit als ,das Forschungsinstrument par excellence, was erfordert, sich als aktiven Teil des Erkenntnisprozesses zu reflektieren" (ebd.).

\subsection{Teilnehmende Beobachtung und Beobachtungsprotokoll}

Trotz der für die Ethnographie als wichtig einzuschätzenden ,methodischen Offenheit“ gehört ,zum festen Kern jeder ethnografischen Studie die Teilnehmende Beobachtung“" (Thomas 2019, S. 3). So besteht die Herausforderung für ethnographisch Forschende darin, „das ,wirkliche“ Leben im Feld erst einmal aufzustöbern, damit in Kontakt zu treten und es sukzessive von innen her kennen zu lernen und zu erschließen“ (ebd.). Dies geschieht nicht ohne eine angemessene Reflexion und eine weitgreifende methodologische Auseinandersetzung im ethnographischen Diskurs. Die methodologische Diskussion über das ethnographische Beobachtungsprotokoll 
bezieht sich etwa auf die Frage nach der konkreten Praxis des Ethnographierens (vgl. Breidenstein et al. 2013), auf die Frage nach dem Wie der Anfertigung des Beobachtungsprotokolls (vgl. Cloos 2010) oder auch auf die Frage des Zusammenspiels von Forschenden und Feld und dessen Implikationen für den Forschungsprozess und die Forschungsergebnisse. Letztere ist nicht nur für die Praxis der teilnehmenden Beobachtung an sich, sondern in besonderem Maße auch für das Verfassen des Beobachtungsprotokolls bedeutsam. Dem Beobachtungsprotokoll kommt in der ethnographischen Forschung die Aufgabe zu, im Forschungsprozess Erlebtes, Gesehenes und Erfasstes festzuhalten. Laut Cloos (2010) ist der „Gesamtprozess des ethnographischen Schreibens [...] eine Kunstlehre, die methodisch kaum zu operationalisieren“ (ebd., S. 182) ist. Zudem macht er deutlich, dass ,die Ethnographie sich von anderen Forschungsstrategien durch einen weniger methodisch strengen Umgang mit den gewonnenen ,Daten “" (ebd.) unterscheidet. Cloos (ebd.) beschreibt weiterhin einen hervortretenden Unterschied der ethnographischen Erhebung zu anderen Forschungsstrategien:

Der wesentliche Unterschied zu anderen Forschungsstrategien scheint zu sein, dass im Rahmen ethnographischer Forschungsstrategien erstens Datenerhebung und Dateninterpretation weniger streng voneinander getrennt werden und zweitens der größere Teil der EthnographInnen sich nicht auf die Protokolle und Feldnotizen als fertige Transkriptionen einer erlebten Praxis verlassen, die es anschließend nur noch zu interpretieren gilt. Die vielschichtige Schreibpraxis bringt stattdessen vielfältige Texte hervor, die durch neue Erfahrungen und Texte ergänzt, moduliert, neu sortiert, reformuliert oder auch vernichtet werden können und abschließend in einer dichten, ethnographischen Beschreibung münden (ebd., S. 181).

Die hier diskutierte Frage nach dem Stellenwert des Protokolltextes zeigt, dass sich die Protokollierung neben der teilnehmenden Beobachtung als weiterer bedeutsamer Aspekt für den ethnographischen Forschungsprozess erweist. Ethnographie versteht sich daran anschließend nicht nur als Methode, ,,sondern auch als ein Produkt von Forschung, indem sie einen Forschungsprozess in seinen einzelnen Schritten dokumentiert" (Wilde 2015, S. 110).

Zur Bestimmbarkeit von Subjektivitäts- und Objektivitätsansprüchen im Beobachtungsprotokoll zeigen Breidenstein und Kelle (1998) anhand eines von ihnen aufgezeichneten Gesprächs zu dieser Frage, welche Fallstricke das Versteifen auf einerseits eher technisierte Protokollstile und andererseits subjektiv gehaltene Beobachtungsprotokolle bereithält. So plädiert die erste Sprecherin dafür, ,gerade die situativen Bewertungen und Gefühle mit zu erheben, denn diese gehören konstitutiv zu den Situationen dazu. Das Geschehen ist ja auch für die Teilnehmer mit Emotionen und Urteilen verknüpft, eine um diese Dimensionen bereinigte Beschreibung entspricht insofern auch nicht dem Erleben von Teilnehmern“ (ebd., S. 145). Andererseits wird als Reaktion darauf hervorgehoben, dass auch die Gegenposition darauf aus ist, nach den „Bewertungen des Geschehens“ (ebd.) durch die feldeigenen Teilnehmenden zu fragen. Jedoch, so stellt dieser Sprecher heraus, sei es wichtig, „um die Gefühle und Bewertungen von Teilnehmern adäquat erfassen zu können, von den eigenen Bewertungen der Situation“ (ebd.) abzusehen und davon Distanz 
zu gewinnen. Es wird verhandelt, welche Instrumente nötig sind, um die dem Feld am nächsten kommende ,dichte Beschreibung“ (Geertz 1987) zu ermöglichen. Die dichte Beschreibung wird somit beschreibbar als „Ausdruck der Vieldeutigkeit, in die sich auch eine naturalistische Ethnographie verstrickt, will sie die praktischen Vollzüge vor Ort und das lokale Wissen der Teilnehmerinnen nachvollziehen“, so Scheffer (2010, S. 141).

\section{Ethnographie als Interaktionsprozess}

Der nun zu unterbreitende Vorschlag einer verstärkten Fokussierung auf die interaktionalen Bezugnahmen im Forschungsprozess knüpft an die voranstehenden Ausführungen an und möchte sich an das Potenzial der Rekonstruktion unterschiedlicher Protokollebenen im Beobachtungsprotokoll herantasten. Es soll untersucht werden, inwiefern die Subjektivität der Beobachterin als Anhaltspunkt für die während der Untersuchung entstehenden Interaktionen methodisch sinnvoll nutzbar gemacht werden kann. Dazu wird ein interpretatives Vorgehen vorgestellt, das den subjektiven Faktor der oder des Forschenden in den Fokus der Interpretation stellt. Es geht darum, im Modus der stellvertretenden Aneignung eine methodologische Grundlage für die Interpretation von Beobachtungsprotokollen zu markieren, der es gelingt, Hinweise auf das Wechselverhältnis von Forschung und Feld innerhalb des ethnographischen Forschungsprozesses zu geben.

\subsection{Ich-Ebenen im Beobachtungsprotokoll}

Statt eines distanziert „stillosen“ (Reichertz 1992, S. 333) Sprachstils des Beobachtungsprotokolls möchten die vorliegenden Überlegungen auf die Vorteile eines stark subjektiv geprägten Schreibstils aufmerksam machen. Dabei wird die Idee verfolgt, dass in Beobachtungsprotokollen drei Ich-Ebenen zu rekonstruieren sind, die im Folgenden dargestellt werden sollen. So finden sich in Beobachtungsprotokollen ein erlebendes Ich, ein protokollierendes Ich und ein im Nachgang reflektierendes Forschergruppen-Ich. Das erlebende Ich spiegelt hauptsächlich die persönlichen Erlebnisse des oder der Forschenden wider. Es geht hierbei um die bewusste Einlassung auf die Gefühle und Emotionen der Autorin oder des Autors des Protokolltextes. Widerstände, Sympathie, Präferenzen und ähnliche affektive Regungen geraten in den Vordergrund. Im Erleben der Autorin oder des Autors sollen im Modus der stellvertretenden Aneignung die Eigenheiten und Besonderheiten des untersuchten Feldes hervorgehoben werden. Mit welchen Barrieren hat die oder der Forschende beim Eintritt ins Forschungsfeld zu kämpfen? Wird die oder der Forschende mit offenen Armen in das Feld aufgenommen? Es geht um die Verschriftlichung der Bewertungen, die auf einer präreflexiven Haltung basieren. Das erlebende Ich beschreibt die Aneignungsweisen von im Feld erlebten Situationen aus der Perspektive einer teilnehmenden Person. So verfügt das erlebende Ich über eine spezifisch subjektive Form des Wissens über das Feld, da dieses ausschließlich über die Teilnahme an dem sich interaktional verfertigenden Erleben innerhalb des Forschungsfeldes zu erlangen ist. 
Die zweite analytische Perspektive ist das das Protokoll verfassende Ich. Während es sich beim erlebenden Ich um eine stark auf die subjektive Haltung der oder des Forschenden ausgerichtete Perspektive handelt, ist die Haltung des das Protokoll verfassenden Ich bereits auf eine Fragestellung bezogen. Es setzt Relevanzen, die im Nachgang schriftlich fixiert werden, und nimmt die forschungsbezogenen Vorüberlegungen zum Anlass, das im Feld Beobachtete unter einer bestimmten Fragestellung bereits vorzusortieren. Im Beobachtungsprotokoll tritt diese Ich-Ebene besonders dann hervor, wenn implizit die Beantwortung einer bestimmten Forschungsfrage vorgenommen wird. So kann diese Ebene bereits als analytische Beobachtungsebene festgehalten werden, die den Modus der affektiven Aneignung bereits hinter sich gelassen hat und stärker auf die Beobachtung bestimmter Ordnungsbildungen fokussiert ist. Zuletzt kann das Forschergruppen-Ich als im Nachgang forschungsbezogen reflektierendes Ich festgehalten werden. Im Forschergruppen-Ich treten distanzierte Beobachtungen und protokollierte Relevanzen in den Vordergrund. Diese nehmen eine stellvertretende Aneignung und Deutung des Geschehenen vor. Diese dritte analytische Perspektive des Protokolls setzt sich künstlich in Distanz zum Beobachteten. Im Nachhinein bezieht sie die erste und zweite Ich-Perspektive in das Beobachtete mit ein und hält diese Sortierung im Hinblick auf die eigene Forschungsfrage und deren Reflexion im Protokolltext fest. Das Forschergruppen-Ich wird dann sichtbar, wenn Kontextwissen hinzugezogen wird oder bereits erste Interpretationen des Erlebten im Horizont der eigenen Forschungsfrage und des Forschungskontextes vorgenommen werden (vgl. Cafantaris 2020, S. 43).

\subsection{Die Rekonstruktion von Subjektivität im hybriden Lernsetting als Forschungsperspektive}

Die oben dargestellten Ich-Ebenen treten nicht etwa linear im Beobachtungsprotokoll auf. Sie sind vielmehr fluider Form, bedingen sich gegenseitig und heben sich an anderer Stelle gegenseitig auf. Die Unterscheidungen der drei Ebenen dient der Komplexitätsreduktion sozialer Wirklichkeit, sollte jedoch nicht kausal, sondern als Instrument der Datensortierung genutzt werden, die an geeigneter Stelle wieder die Brüchigkeit sozialer Interaktionen berücksichtigt. An der nun folgenden Fallinterpretation soll gezeigt werden, wie die Protokollantin das von ihr untersuchte Feld zunächst durch die Versprachlichung von Sortierungsbewegungen (Ich-Ebenen im Beobachtungsprotokoll) zu ordnen sucht und die interaktionalen Bezugsnahmen forschungsbezogen rekonstruiert.

Das vorliegende Beobachtungsprotokoll ist der Studie Umgang mit Wissen in der Kulturarbeit für Erwachsene. Pädagogische Kommunikation in hybriden Settings der Erwachsenenbildung (Cafantaris 2020) entnommen. In der Studie wurde ein von einer Stiftung ausgerichtetes Geschichtsprojekt für Erwachsene auf die Frage hin untersucht, welche pädagogisch und nichtpädagogisch strukturierten Formbildungen zu beobachten sind und inwiefern sich diese Merkmale als spezifisch für ein hybrides Lernsetting erweisen. Der theoretische Bezug der Studie lag dabei hauptsächlich in einem systemtheoretisch modellierten Zugang, der die Rekonstrukti- 
on von Kommunikationsformaten (pädagogische Kommunikation ${ }^{2}$, Wissensvermittlung, gesellige Kommunikation) in den Fokus der Erhebung stellte. Das beforschte Projekt wurde von einer renommierten Bürgerstiftung ausgerichtet und ermöglichte 25 Teilnehmerinnen und Teilnehmern die intensive Auseinandersetzung mit einem stadtteilhistorischen Gegenstand. Während der 18-monatigen Projektlaufzeit wurden die Teilnehmenden durch eine eng geknüpfte Projektstruktur, einen Projektkoordinator und eine finanzielle Unterstützung begleitet. Das Projektziel bestand darin ein repräsentatives Endprodukt zu erstellen, welches das Ergebnis der Arbeit und Recherchen innerhalb des Projekts abbilden sollte. Die Teilnehmenden fanden sich in drei unterschiedlichen Veranstaltungsformaten zusammen: der Staffelübergabe als Initiations- und Verabschiedungsritual, dem Werkstatt-Treffen als wissensvermittlungsbezogenem Workshop und den informellen Treffen, die einmal pro Monat den regelmäßigen Austausch über die jeweiligen Projekte ermöglichen sollten. Der exemplarisch gewählte Ausschnitt aus dem vorliegenden Beobachtungsprotokoll protokolliert eine Szene aus einem sogenannten informellen Treffen des untersuchten Geschichtsprojekts. Anhand der Fallvignette soll erstens die Analyse der drei IchEbenen veranschaulicht werden. Daran anknüpfend wird zweitens das besondere Wechselverhältnis von Forscherin und Teilnehmer veranschaulicht, welches die Rekonstruktion der feldeigenen Logik des Geschichtsprojekts aufzuschlüsseln vermag.

\subsubsection{Fallvignette: Protokolltext}

„Ich komme pünktlich um 19:00 Uhr im Haus des Geschichtsvereins [Name des Stadtteils] an, in dem heute das informelle Treffen der [Name des Projekts] stattfindet. Es handelt sich um ein Haus, welches zunächst eher an ein Wohnhaus erinnert. Erst beim Eintreten in die Räumlichkeiten wird sichtbar, dass es sich hier um einen Geschichtsverein handeln könnte. In der Mitte des Raumes steht ein großer, viereckiger Tisch. Um ihn herum wurden Stühle gestellt. An den Wänden des Raumes hängen Bilder, die an die vergangene Zeit erinnern, an einer der Wände hängt ein großes Schild mit der Aufschrift ,[Name des Stadtteils]' [...]. Es ist an der einen Seite abgebrochen und ich vermute, es ist ein Schild, welches am alten [Name des Stadtteils] Bahnhof hing, bevor dieser umgebaut wurde. Der Raum ist zu dem Zeitpunkt meiner Ankunft schon reichlich mit Personen gefüllt. Sie scheinen sich angeregt miteinander zu unterhalten. Ich suche nach bekannten Gesichtern und es irritiert mich, dass drei bis vier mir unbekannte Personen in dem Raum anwesend sind. Eine dieser Personen wird mir von [Name], einer Hobby-Historikerin, mit der ich Interviews führe und die ich daher auch schon etwas besser kenne, vorgestellt. Es ist die Vorsitzende des Geschichtsvereins, ehemalige Hobby-Historikerin und - so fällt es mir im Nachhinein ein - die Frau, die auf der aktuellen Broschüre des Projekts ,GeschichteErforschen “ abgebildet ist. Wir stellen uns gegenseitig vor und ich gehe weiter in den Raum hinein, wo ich sogleich TN2, auch einer meiner Interviewpartner, sehe. Wir haben uns lange nicht gesehen und ohne lange

2 Zum Begriff der pädagogischen Kommunikation vgl. Kade (2004; 2017). 
darüber nachzudenken, umarmen wir uns freundschaftlich. Ich freue mich einerseits, andererseits ist mir die freundschaftliche Begrüßung unangenehm, da ich denke, dass TN2 mich in erster Linie als so etwas wie eine ,Verbündete unter ,älteren Menschen ' begreift und mich nicht als Forscherin wahrnimmt. Ich übergehe meine Unsicherheit und frage ihn nach seinem Projekt. Er berichtet, dass er eine Homepage und zusätzlich einen Wikipedia-Eintrag zu seinem Thema erstellt habe. Der Wikipedia-Eintrag habe durch ,Connections " schon in den ersten Tagen eine sehr hohe Anzahl an Klicks erreicht. Er wirkt sehr zufrieden damit. Dann frage ich, ob er nicht auch ein Buch geplant hatte. Ja, das sei in Arbeit, antwortet er auf meine Frage hin. Aber der Termin der Fertigstellung des Buches sei auf das nächste Jahr verschoben worden. Ich hake noch einmal nach und frage, ob dann die Homepage und der Wikipedia-Eintrag sein Endprodukt für das Projekt seien, und er beantwortet meine Frage mit Ja. AuBerdem hätte der Projektkoordinator an ihn herangetragen, dass er noch einen Vortrag halten solle. Im weiteren Gespräch geht es darum, wo dieser Vortrag stattfinden könnte, und ich schlage ein Restaurant/Veranstaltungsort im [Name des Stadtteils] vor“" (Cafantaris 2020, S. 136f.).

\subsubsection{Fallvignette: Interpretation}

Der Protokollausschnitt markiert zunächst die pünktliche Ankunft des protokollierenden Ich im Untersuchungsfeld. Ebenso wird ein bereits vorbereitetes Setting beschrieben: Die Angabe einer vereinbarten Uhrzeit, die bereits vorhandene Bestuhlung des Ortes und der Verweis auf eine Gruppe bereits anwesender Personen lässt auf ein, zu einem bestimmten Zweck, vereinbartes Treffen schließen. Es werden interne Suchbewegungen des Ich beschrieben, die sich vor allem in der Unterscheidung von bekannten und unbekannten Personen äußern. Die erste Interaktion mit einer bekannten Person führt zu einer weiteren Erläuterung des Kontextes, der als von Hobby-Historikern besucht und mit der Nennung des Projekttitels „GeschichteErforschen“ genauer bestimmt wird. Die Bedeutungszuweisung gegenüber der räumlichen Vorstrukturierung und der durch die Protokollantin angeführten Bezeichnungen (Hobby-Historiker und Projekttitel), sowie der Verweis auf eine bereits bestehende Broschüre erzeugen den Eindruck eines bereits institutionalisierten Settings, welches den Umgang mit Wissen zum Thema Geschichte prozessiert.

Während die erste Begegnung mit einer bekannten Person im Feld eher höflichformal vollzogen wird, kommt es bei der zweiten Begegnung zu einem inneren Aushandlungsprozess der erlebenden Protokollantin. Durch das Aufzeigen einer affektiven Handlung, einer Umarmung, wird das Aufeinandertreffen der beiden Personen als informell beschreibbar. Die Umarmung wird im Folgenden gleichsam noch einmal überdacht und aus der Bewertung des erlebenden Ich als der Situation unangemessen kategorisiert. So wird die Umarmung unmittelbar als zu privat eingestuft. Die Forscherin bemüht sich in der Folge, kommunikativ wieder in ein spezifisches - hier professionelles - Verhältnis zu ihrem Gesprächspartner zu treten. Der körperliche Kontakt erscheint im Vergleich zu dem höflich-formalen Aufeinandertreffen mit der Interviewpartnerin in der vorangegangenen Sequenz also erstens different, 
zweitens erscheint er mit Blick auf den Kontext als - im Erleben der Forscherin unangemessen. Veranschaulicht wird dies am protokollierten Gedankengang der Beobachterin. In ihrer Beschreibung führt sie aus, dass sie zwar ihrem ersten Impuls folgt und die Person umarmt, sich jedoch quasi simultan daran erinnert, dass dies ihrer Rolle als distanzierter Beobachterin nicht entspricht. Durch die freundschaftliche Umarmung des Teilnehmers wird der Forscherin also für diesen kurzen Moment der Status als Forscherin abgesprochen und sie kommt in eine krisenhafte Situation, denn ihre Rolle im Feld wird für einen kurzen Moment unklar. Die Perspektive wechselt damit stärker in die Introspektion der Forscherin. Das erlebende Ich kommt zum Tragen: So zeigt sich in der Reaktion der Protokollantin eine Variante, ihre Rolle im Feld abermals zu sichern. Das Gespräch wird durch die Thematisierung des Projekts auf eine sachliche Ebene gebracht. Von der freundschaftlich-diffusen Kommunikation, die die Praktik des Umarmens miteinbezieht, wird die Kommunikation themenzentriert auf das Geschichtsprojekt des Teilnehmers gelenkt. Im weiteren Verlauf des Protokollausschnitts wird beschrieben, wie der Teilnehmer bereitwillig über die neuesten Entwicklungen seines Blogs und des von ihm verfassten Eintrags auf einer Online-Plattform berichtet. Dieser habe innerhalb kurzer Zeit Erfolg in Form von Klicks erlangt, was der Teilnehmer „Connections“, also Verbindungen, zuschreibt, die er jedoch nicht weiter erläutert. Die Sprache, die der Teilnehmer hierzu verwendet, lässt ebenso wie die informelle Umarmung auf einen informell anmutenden Sprachgebrauch schließen, wie er unter Personen gleichen Alters und aus ähnlichen kulturellen bzw. sozialen Kontexten üblich ist. In jedem Fall verweist der Anglizismus ,connections“ auf einen anderen Referenzrahmen als den des Geschichtsprojekts. Über ein soziales Netzwerk zu verfügen, kann auch für die Arbeit an einem historischen Projekt von Nutzen sein, dies aber nur flankierend, da es in der Auseinandersetzung mit einem historischen Projekt vielmehr um einen intrinsischen, auf einen subjektiven Bildungsprozess ausgerichteten Vorgang geht. Dennoch erscheint es so, als würde der Teilnehmer davon ausgehen, dass die Protokollantin den gewählten Referenzrahmen versteht. Er adressiert sie nicht so sehr als Forscherin, sondern eher als Peer und verwendet eine Sprache, die im Feld sonst nicht als üblich erscheint. Indem die Protokollantin sich anschließend verstärkt Fragen nach dem Endprodukt des Teilnehmers zuwendet, nimmt sie diese Form des Gesprächsangebots nicht an. Nimmt man den Schreibstil des Protokolls näher in den Blick, kommt es in dem Protokollausschnitt zu einer thematischen Zuspitzung: Es geht verstärkt um das Endprodukt des Teilnehmers. Während sich das protokollierende Ich nach der konkreten formalen Gestaltung dieses Endprodukts erkundigt, erscheinen die Antworten des Teilnehmers weniger konkret, als die Protokollantin es zu erwarten scheint. Sie fragt indessen sehr präzise nach dem Endprodukt, das als „Buch“ begrifflich festgelegt wird. Das Buch, das im Gegensatz zu den neuen Medien einen klassischen und zudem bildungsorientierten Gegenstand darstellt, wird damit von der Beobachterin als präferiertes Medium markiert. Sowohl in der spezifischen Hervorhebung der Erwartung, ein Buch anzufertigen, als auch in der thematisch verdichteten Abfrage zur konkreten Anfertigung eines Endprodukts, legt das Gespräch eine Erwartungshaltung nahe, die auf eine Leistungsdimension, im Sinne der Überprüfung der Wissensaneignung, im Geschichtsprojekt schließen lässt. Die Protokollantin übernimmt stellvertretend für die Stiftung die Abfrage des 
jeweiligen Arbeitsstands und ko-produziert gleichsam den Leistungs- und Erwartungshorizont innerhalb des hybriden Lernsettings. Obgleich der Anfang des Protokolls die gesellige Dimension des Aufeinandertreffens betont und ausschließlich die räumlichen Bedingungen auf eine thematische Zentrierung hinweisen, gewinnt die soziale Ordnung innerhalb der Interaktion zwischen Forscherin und Teilnehmer einen spezifischen Aneignungsbezug, der sich in der Zuspitzung der Erwartung an das Endprodukt zeigt. Neben der diffusen Zusammenkunft von an Geschichte interessierten Erwachsenen entsteht über die Bezugnahme auf einen Gegenstand, der die Wissensaneignung des Teilnehmers ebenso dokumentieren als auch präsentieren soll, demzufolge eine normative Erwartung an Bedeutung, die zur interaktionalen Stabilisierung des hybrid-pädagogisch strukturierten Lernsettings beiträgt (vgl. Cafantaris 2020, S. $137 \mathrm{f}$.).

\section{Diskussion}

Die Fallvignette veranschaulicht vor allem zwei zentrale methodologische Herausforderungen der ethnographischen Erforschung hybrider Lernsettings: erstens das Verhältnis von Diffusion und Stabilisierung und zweitens den subjektiven Faktor ethnographischer Forschung. Mit Blick auf das Verhältnis von Diffusion und Stabilisierung und in Rekurs auf die Forschung zu hybriden Lernsettings verdeutlicht die Fallvignette das Changieren der Forscherin-Feld-Interaktion zwischen einer geringen und einer starken Themenzentrierung: So wird erkennbar, dass sich in der protokollierten Interaktion eine bestimmte Aneignungserwartung aufseiten der Beobachterin durchsetzt, die der Interaktion ein pädagogisch strukturiertes Gewand anlegt. Die in der Interaktion zutage tretende Gleichzeitigkeit von Produktorientierung aufseiten der Beobachterin und Aneignungsautonomie aufseiten des Teilnehmers verweist auf das zentrale Merkmal hybrider Lernsettings, dass mehrere Möglichkeiten der Bezugnahme auf den Umgang mit Wissen oder Nichtwissen gleichsam ko-existieren können, denn die pädagogische Formbildung bleibt ungewiss und changiert zwischen ,aneignungsbezogener Wissenskommunikation“ (Kade 2017, S. 6) und freigegebener Aneignung (vgl. ebd.).

Mit Blick auf den zweiten Aspekt, den subjektiven Faktor ethnographischer Forschung, muss zunächst festgehalten werden, dass die Stärke der ethnographischen Forschungshaltung neben dem geschulten Beobachten insbesondere im Erleben der Prozesse des Untersuchungsfeldes und der Erschließung der feldeigenen Logik liegt. Dies unterscheidet die Methode von technisierten Forschungsmethoden, deren Ziel die möglichst ,realitätsnahe“ Abbildung von Wirklichkeit ist. Die ethnographische Beobachterin ist im Unterschied dazu daran interessiert, die feldeigenen Relevanzsetzungen zu erschließen und diese im Beobachtungsprotokoll festzuhalten, um sie im Nachgang methodisch kontrolliert auswerten zu können. Im Zusammenspiel von Forschenden und Feld entstehen im besten Fall interaktionale Bezugnahmen, die sich als Aushandlungsprozesse verstehen lassen und die sich sowohl in Form der Öffnung als auch in Form der Schließung gegenüber von Forschung zeigen können. Diese Verhältnisbestimmungen geben Hinweise auf die jeweiligen Strukturlogiken des Feldes. Sie erleichtern damit die Schwerpunktsetzung im weiteren Forschungs- 
prozess und die theoretische Rückbindung. Was bedeuten diese Überlegungen nun in der Gesamtschau für die weitere ethnographische Erforschung hybrider Lernsettings? Deutlich wird, dass die Erforschung hybrider Lern- und Bildungsorte einer starken Anbindung der Forschenden an das Feld bedarf, die ein möglichst unmittelbares Erleben der feldeigenen Prozesse mit sich bringt. So kann es gelingen, diffundierende von formal-strukturierten Elementen der Interaktion zu unterscheiden und für die analytische Untersuchung rekonstruierbar zu machen. Methodologische Überlegungen wie die in diesem Beitrag vorgestellten unterschiedlichen IchEbenen des Beobachtungsprotokolls sind eine Variante der nachträglichen Reflexion der eigenen Forschungstätigkeit. Im Zusammenspiel von theoretischer Rückbindung und methodologisch angebundener Vorgehensweise kann soziale Wirklichkeit somit ähnlich einer Mosaikstruktur entschlüsselt werden. Damit untersuchbar werden die komplexen Formen des Verhältnisses von vermittlungs- und aneignungsbezogenen Sequenzen und der Sequenzen innerhalb hybrider Lernsettings die Aneignung freisetzen, diese also nicht überprüfen und thematisieren.

Open Access Dieser Artikel wird unter der Creative Commons Namensnennung 4.0 International Lizenz veröffentlicht, welche die Nutzung, Vervielfältigung, Bearbeitung, Verbreitung und Wiedergabe in jeglichem Medium und Format erlaubt, sofern Sie den/die ursprünglichen Autor(en) und die Quelle ordnungsgemäß nennen, einen Link zur Creative Commons Lizenz beifügen und angeben, ob Änderungen vorgenommen wurden.

Die in diesem Artikel enthaltenen Bilder und sonstiges Drittmaterial unterliegen ebenfalls der genannten Creative Commons Lizenz, sofern sich aus der Abbildungslegende nichts anderes ergibt. Sofern das betreffende Material nicht unter der genannten Creative Commons Lizenz steht und die betreffende Handlung nicht nach gesetzlichen Vorschriften erlaubt ist, ist für die oben aufgeführten Weiterverwendungen des Materials die Einwilligung des jeweiligen Rechteinhabers einzuholen.

Weitere Details zur Lizenz entnehmen Sie bitte der Lizenzinformation auf http://creativecommons.org/ licenses/by/4.0/deed.de.

\section{Literatur}

\section{Verwendete Literatur}

Amann, K. \& Hirschauer, S. (1997). Die Befremdung der eigenen Kultur. Zur ethnographischen Herausforderung soziologischer Empirie. Frankfurt a. M.: Suhrkamp.

Bock, K. (2010). Feldnotizen über das Zustandekommen von Gesprächen mit Kindern oder: Die Ethnographin im Kinderbett. In F. Heinzel, W. Thole, P. Cloos \& S. Köngeter (Hrsg.), „Auf unsicherem Terrain“. Ethnographische Forschung im Kontext des Bildungs- und Sozialwesens (S. 85-93). Wiesbaden: Springer VS.

Bollig, S. (2010). Ja, ist das jetzt mehr ein Praktikum, oder was? In F. Heinzel, W. Thole, P. Cloos \& S. Köngeter (Hrsg.), „Auf unsicherem Terrain“. Ethnographische Forschung im Kontext des Bildungs- und Sozialwesens (S. 107-116). Wiesbaden: Springer VS.

Breidenstein, G. (2017). Interdisziplinäre Tradition und disziplinäre Konvention in der erziehungswissenschaftlichen Ethnographie. Zeitschrift für Qualitative Forschung, 18(1), 9-20.

Breidenstein, G., \& Kelle, H. (1998). Geschlechteralltag in der Schulklasse. Weinheim: Juventa.

Breidenstein, G., Hirschauer, S., Kalthoff, H., \& Nieswand, B. (2013). Ethnografie. Die Praxis der Feldforschung. Stuttgart: UTB.

Bühler-Niederberger, D. (2010). Die Beobachtung der EthnographInnen - kommentierende Notizen. In F. Heinzel, W. Thole, P. Cloos \& S. Köngeter (Hrsg.), „Auf unsicherem Terrain “. Ethnographische Forschung im Kontext des Bildungs- und Sozialwesens (S. 276-272). Wiesbaden: Springer VS. 
Cafantaris, K. (2020). Umgang mit Wissen in der Kulturarbeit für Erwachsene. Pädagogische Kommunikation in hybriden Settings der Erwachsenenbildung. Wiesbaden: Springer VS.

Cloos, P. (2010). Narrative Beobachtungsprotokolle. In F. Heinzel, W. Thole, P. Cloos \& S. Köngeter (Hrsg.), „Auf unsicherem Terrain“. Ethnographische Forschung im Kontext des Bildungs- und Sozialwesens (S. 181-191). Wiesbaden: Springer VS.

Cloos, P., \& Thole, W. (Hrsg.). (2006). Ethnografische Zugänge. Professions- und adressatInnenbezogene Forschung im Kontext von Pädagogik. Wiesbaden: Springer VS.

Dinkelaker, J. (2008): Kommunikation von (Nicht-)Wissen. Eine Fallstudie zum Lernen Erwachsener in hybriden Settings. Wiesbaden: Springer VS.

Dinkelaker, J. (2018). Lernen Erwachsener. Stuttgart: Kohlhammer.

Fauré, E., et al. (1973): Wie wir leben lernen. Der UNESCO-Bericht über Ziele und Zukunft unserer Erziehungsprogramme. Reinbek bei Hamburg: Rowohlt.

Geertz, C. (1987). Dichte Beschreibung. Beiträge zum Verstehen kultureller Systeme. Frankfurt a. M.: Suhrkamp.

Heinzel, F. (2010). Ethnographische Untersuchung von Mikroprozessen in der Schule. In F. Heinzel, W. Thole, P. Cloos \& S. Köngeter (Hrsg.), „Auf unsicherem Terrain“. Ethnographische Forschung im Kontext des Bildungs- und Sozialwesens (S. 29-47). Wiesbaden: Springer VS.

Heinzel, F., Thole, W., Cloos, P., \& Köngeter, S. (Hrsg.). (2010). „Auf unsicherem Terrain“. Ethnographische Forschung im Kontext des Bildungs- und Sozialwesens. Wiesbaden: Springer VS.

Hünersdorf, B. (2009). Der klinische Blick in der sozialen Arbeit. Systemtheoretische Annäherungen an eine Reflexionstheorie des Hilfesystems. Wiesbaden: Springer VS.

Kade, J. (2004). Erziehung als pädagogische Kommunikation. In D. Lenzen (Hrsg.), Irritationen des Erziehungssystems. Pädagogische Resonanzen auf die Systemtheorie (S. 199-232). Frankfurt a. M.: Suhrkamp.

Kade, J. (2017). Subjektivierungsformen und Subjektivierungspraktiken. Zur Ordnung des Pädagogischen diesseits und jenseits der Schule. EEO - Enzyklopädie Erziehungswissenschaft Online. Weinheim: Beltz Juventa.

Lindner, R. (1981). Die Angst des Forschers vor dem Feld. Überlegungen zur teilnehmenden Beobachtung als Interaktionsprozeß. Zeitschrift für Volkskunde, 77, 51-66.

Lüders, C., Kade, J., \& Hornstein, W. (2004). Entgrenzung des Pädagogischen. In H.-H. Krüger \& W. Helsper (Hrsg.), Einführung in Grundbegriffe und Grundfragen der Erziehungswissenschaft (6. Aufl., S. 223-232). Wiesbaden: Springer VS.

Reichertz, J. (1992). Beschreiben oder Zeigen - über das Verfassen ethnographischer Berichte. Soziale Welt, 3(43), 331-350.

Scheffer, T. (2010). Ethnographie mit System am Beispiel von englischen Strafverfahren. In R. John, A. Henkel \& J. Rückert-John (Hrsg.), Methodologien des Systems: Wie kommt man zum Fall und wie dahinter? (S. 141-158). Wiesbaden: Springer VS.

Seitter, W. (1999). Riskante Übergänge in der Moderne. Vereinskulturen Bildungsbiographien, Migranten. Wiesbaden: Springer VS.

Seitter, W. (2000). Lesen, Vereinsmeiern, Reisen. (Vergessene) Elemente einer Theorie lebenslangen Lernens. Zeitschrift für Pädagogik, 1(46), 81-96.

Seitter, W. (2011). Pädagogische Felder. In J. Kade, W. Helsper, C. Lüders, B. Egloff, F.-O. Radtke \& W. Thole (Hrsg.), Pädagogisches Wissen. Erziehungswissenschaft in Grundbegriffen (S. 87-92). Stuttgart: Kohlhammer.

Thole, W., Heinzel, F., Cloos, P. \& Köngeter, S. (2010): „Auf unsicherem Terrain“. Ethnographische Forschung im Kontext des Bildungs- und Sozialwesens. In F. Heinzel, W. Thole, P. Cloos \& S. Köngeter (Hrsg.) „Auf unsicherem Terrain“. Ethnographische Forschung im Kontext des Bildungs- und Sozialwesens (S. 11-13). Wiesbaden: Springer VS.

Thomas, S. (2019). Ethnografie. Qualitative Sozialforschung. Wiesbaden: Springer VS.

Wiesemann, J. (2010). Ethnographie (machen) mit Kindern in der Schule: Die Beobachtung der Beobachter. In F. Heinzel, W. Thole, P. Cloos \& S. Köngeter (Hrsg.), „Auf unsicherem Terrain“. Ethnographische Forschung im Kontext des Bildungs- und Sozialwesens (S. 143-169). Wiesbaden: Springer VS.

Wilde, D. (2015). Dinge sammeln. Annäherung an eine Kulturtechnik. Bielefeld: transcript.

Wischmann, A. (2017). Dimensionen des Lernens und der Bildung. Konturen einer kritischen Lern- und Bildungsforschung entlang einer Reflexion des Informellen. Weinheim und Basel: Beltz Juventa.

Wolff, S. (2000). Wege ins Feld und ihre Varianten. In U. Flick, E. von Kardoff \& I. Steinke (Hrsg.), Qualitative Forschung (S. 334-345). Reinbek bei Hamburg: Rowohlt. 


\section{Weiterführende Literatur}

Thole, W. (2010). Ethnographie des Pädagogischen. In F. Heinzel, W. Thole, P. Cloos \& S. Köngeter (Hrsg.), „Auf unsicherem Terrain“. Ethnographische Forschung im Kontext des Bildungs- und Sozialwesens (S. 17-38). Wiesbaden: Springer VS.

Publisher's Note Springer Nature remains neutral with regard to jurisdictional claims in published maps and institutional affiliations. 